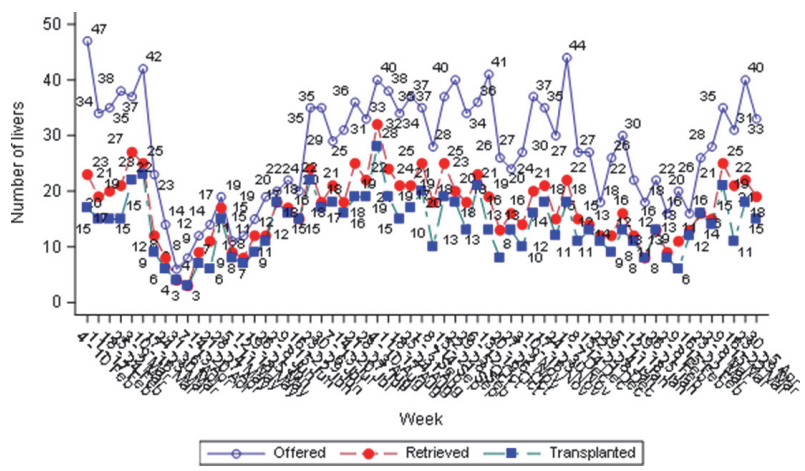

Abstract P091 Figure 1 Number of livers from UK deceased doners offered, retrieved and transplanted, 4 February 2020 to 5 April 2021

NHSE and some patient care was transferred between centres, when overwhelmed critical care necessitated temporary unit closures.

Results During 2020/21, there was a significant fall in the total number of annual liver donors (21\%; 870 from 1088) and transplants (22\% 749 from 950) compared to 5-year mean, prior to the pandemic. This was exclusively amongst adult recipients $(23 \% ; 672$, vs 868$)$, both elective $(22 \% ; 609$ vs 778$)$ and SU (30\%; 63 vs 90), whilst paediatric activity was maintained (77 vs 81$)$. The reduction in adult LT varied widely geographically (-3 to $-43 \%)$. LT registrations fell $(11 \%$; 1063 vs 1208), again with wide geographic variation (0 to 24\%).

During the 'first wave', we successfully prioritised those with highest illness severity with no reduction in $90 \mathrm{~d}$ patient $(p=0.89)$ or graft survival $(p=0.98)$. There was a small $(5 \%$ cf $3 \%)$ but significant $(p=0.0015)$ increase in deaths/removals from the UK LT waitlist, during this time. During the 'second wave', 5 adult units temporarily closed at various times, whilst 3 'protected' LT centres were maintained at any time. Consequently, 25 waitlist registrants were transferred to protected centres with 10 undergoing LT outwith their original centre.

Discussion A sophisticated national response has maintained a safe and effective UK LT program throughout the first year of COVID. We adapted our resources, implementing phased donor restrictions and a new category for recipient prioritisation. Patients benefitted from collaborative working, enabling those in most need to be transferred and transplanted in protected centres. Consequently, we mitigated against a significant fall in LT activity. Our collaborative response serves as an as exemplar for other specialist healthcare services.

\section{P092 SOCIOECONOMIC STATUS IS NOT A BARRIER TO LIVER TRANSPLANTATION FOR PATIENTS WITH ALCOHOL RELATED LIVER DISEASE}

\footnotetext{
1,2 Harry Spiers*, ${ }^{3}$ Hazel Woodland, ${ }^{1}$ Clare Wai, ${ }^{2}$ Adil Lakha, ${ }^{1,2}$ Paul Gibbs, 1,2Michael Allison. 'The Roy Calne Transplant Unit, Addenbrooke's Hospital, Cambridge, UK; ${ }^{2}$ University of Cambridge, Cambridge, UK; ${ }^{3}$ University Hospitals Southampton NHS Foundation Trust, Southampton, UK
}

\subsection{6/gutjil-2021-BASL.100}

Introduction Alcohol related liver disease (ArLD) carries significant morbidity and mortality; approximately $75 \%$ of deaths from liver disease in the UK result from excess alcohol consumption. Liver disease prevalence shows marked geographical variation and people with liver disease living in the most deprived quintile die on average 9 years earlier than those living in the least deprived quintile. This study reviewed records for patients referred for transplant assessment with a history of ArLD to examine for obvious demographic, social or aetiology-based discrepancies in listing decisions.

Methods Electronic records of patients with a history of ArLD referred to our unit for liver transplant assessment between January 2019 and December 2021 were retrospectively

Abstract P092 Table 1 Baseline demographics, social factors, aetiology of liver disease, period of abstinence and assessment outcome of patients referred for liver transplant assessment with a history of ArLD

\begin{tabular}{|c|c|c|c|}
\hline & $\begin{array}{l}\text { Number assessed } \\
(\%)\end{array}$ & $\begin{array}{l}\text { Number } \\
\text { listed }\end{array}$ & $\begin{array}{l}\% \text { of category } \\
\text { listed }\end{array}$ \\
\hline \multicolumn{4}{|l|}{$\begin{array}{l}\text { Location at time of } \\
\text { assessment }\end{array}$} \\
\hline Inpatient & $50(40)$ & 33 & 66 \\
\hline Outpatient & $75(60)$ & 54 & 72 \\
\hline \multicolumn{4}{|l|}{ Sex } \\
\hline Male & $97(78)$ & 69 & 71 \\
\hline Female & $28(22)$ & 18 & 64 \\
\hline \multicolumn{4}{|l|}{ Age (years) } \\
\hline $20-29$ & $2(2)$ & 1 & 50 \\
\hline 30-39 & $6(6)$ & 4 & 67 \\
\hline $40-49$ & $22(22)$ & 15 & 68 \\
\hline $50-59$ & $49(39)$ & 37 & 76 \\
\hline 60-69 & $43(34)$ & 29 & 67 \\
\hline 70-79 & $3(2)$ & 1 & 33 \\
\hline \multicolumn{4}{|l|}{ IMD Quintile } \\
\hline 1 (most deprived) & $21(17)$ & 13 & 62 \\
\hline 2 & $29(23)$ & 23 & 79 \\
\hline 3 & $33(26)$ & 23 & 70 \\
\hline 4 & $23(18)$ & 16 & 70 \\
\hline 5 (least deprived) & $19(15)$ & 12 & 63 \\
\hline \multicolumn{4}{|l|}{ Residence } \\
\hline Own house & $54(43)$ & 37 & 69 \\
\hline $\begin{array}{l}\text { HA/council house/HMO/ } \\
\text { rehab }\end{array}$ & $22(18)$ & 12 & 55 \\
\hline Rented house & $16(13)$ & 11 & 69 \\
\hline Lives with family & $10(8)$ & 9 & 90 \\
\hline Caravan & $2(2)$ & 2 & 100 \\
\hline Care home & 1 (1) & 1 & 100 \\
\hline Unknown & $20(16)$ & 15 & 75 \\
\hline \multicolumn{4}{|l|}{ Employment } \\
\hline Active & $27(22)$ & 18 & 67 \\
\hline Retired & $21(17)$ & 14 & 67 \\
\hline Unemployed & $77(62)$ & 55 & 71 \\
\hline \multicolumn{4}{|l|}{ Aetiology of liver disease } \\
\hline ARLD & $63(50)$ & 46 & 73 \\
\hline ArLD + HCC & $27(22)$ & 16 & 59 \\
\hline ArLD + 2nd aetiology & $35(28)$ & 25 & 71 \\
\hline \multicolumn{4}{|l|}{ Period of abstinence } \\
\hline $0-3$ months & $15(12)$ & 9 & 60 \\
\hline 4-6 months & $26(21)$ & 18 & 69 \\
\hline 7-12 months & $37(30)$ & 25 & 68 \\
\hline 13-24 months & $25(20)$ & 18 & 72 \\
\hline 25-36 months & $9(7)$ & 7 & 78 \\
\hline$>36$ months & $13(10)$ & 10 & 77 \\
\hline
\end{tabular}


reviewed. Data were collected included baseline demographics, social factors including index of multiple deprivation (IMD), aetiology of liver disease, period of abstinence and assessment outcome (table 1).

Results 125 patients with a history of ArLD were referred for transplant assessment over the 3-year period. The majority (78\%) were male and most (39\%) were 50-59 years. Patients living in the most deprived quintile comprised just $17 \%$ of the group. In 50\% ArLD was the only aetiology listed, 27\% of patients also had hepatocellular carcinoma (HCC) and 28\% had a second, non-malignant, aetiology listed. Patients were divided into groups based on demographics, social factors, aetiology and period of abstinence and the outcome of the transplant assessment was recorded for each group. Table 1 illustrates the percentage of patients in each group listed for transplant. Psychological, social or substance misuse issues (including smoking) were cited as concern for listing in just 7 (5\%) of cases.

Conclusion Our data demonstrates that in patients with a history of ArLD referred to this unit for transplant assessment, there is no listing bias based on socioeconomic background. However, whilst ArLD occurs more often in lower socioeconomic groups, patients from IMD quintile 1 comprised just $17 \%$ of those referred, suggesting a potential referral bias. There was no clear preference for listing patients who had a second causal aetiology for their liver disease. Very few patients were not listed based purely on social/psychological factors. This data should encourage clinicians to refer patients with a history of ArLD for transplant assessment, regardless of their socioeconomic background.

\section{P093 TOLL-LIKE RECEPTOR 4 INHIBITION ACTS SYNERGISTICALLY WITH G-CSF TO PREVENT ORGAN INJURY AND INDUCE LIVER REGENERATION IN ACUTE- ON-CHRONIC LIVER FAILURE}

Cornelius Engelmann, Fausto Andreola*, Abeba Habtesion, Simone Novelli, Annarein JC Kerbert, Nathan Davies, Sofia Ferreira-Gonzalez, Stuart Forbes, Thomas Berg, Rajiv Jalan. Ildh - University College London; Charite - Universitaetsmedizin Berlin, Germany

\subsection{6/gutjnl-2021-BASL.101}

Background and Aims Acute-on-chronic liver failure (ACLF) is characterised by lack of regeneration. Granulocyte colony stimulating factor (G-CSF) carries pro-regenerative properties and has been shown to be of benefit in ACLF. However, the large trial of G-CSF (GRAFT study) in patients with ACLF showed no benefit and in certain groups mortality tended to be higher. This study was performed to define the mechanisms underlying the negative effect of G-CSF and determine whether its beneficial effect could be harnessed using a tolllike receptor 4 (TLR4) antagonist.

Method Two mouse models of ACLF were used: CCL4 $(0.5 \mathrm{mg} / \mathrm{ml}, 6 \mathrm{w})$ to induce chronic liver injury followed by LPS i.p. (Klebsiella, $4 \mathrm{mg} / \mathrm{kg})(\mathrm{n}=4-10)$ or Galactosamine (GalN) i. p. $(1000 \mathrm{mg} / \mathrm{kg})$ as a second hit $(\mathrm{n}=8)$. $1 \mathrm{~h}$ after, G-CSF $250 \mu \mathrm{g} / \mathrm{kg}$ s.c. and/or TLR4-inhibitor TAK-242 $10 \mathrm{mg} / \mathrm{kg}$ i.p. were injected and continued every $24 \mathrm{~h}$. The treatment duration was $24 \mathrm{~h}$ and $5 \mathrm{~d}$ in the LPS model and $48 \mathrm{~h}$ in the GalN model. Samples were stored and analysed for liver injury, inflammation, senescence and regeneration.

Results 6w CCL4 led to bridging fibrosis, TLR4 up-regulation and infiltration of G-CSFr expressing cells. LPS increased ALT levels, cell death $(\mathrm{TUNEL}+)$, enhanced hepatic infiltration of neutrophils (Ly6G+), macrophages $(\mathrm{F} 4 / 80+)$ and TNFa. GCSF increased the $48 \mathrm{~h}$ mortality from $0 \%$ to $66 \%$, aggravated liver inflammation with macrophage and NK cell (CD45+, CD49b+,CD3-,CD19-) infiltration and IL6 expression. G-CSF +TAK-242 reduced the mortality to $0 \%$, abrogated the liver injury (TUNEL) and liver inflammation (macrophages, neutrophils, TNFa, IL6) significantly. In the second model, GalN also induced a significant liver injury. Treatment with G-CSF +TAK-242 was significantly more effective than the individual therapies. G-CSF+TAK-242 was associated with increased liver regeneration evidenced by increased tissue expression of pSTAT3 and BCL2. CCL4+LPS induced a p53 and p16dependent cell cycle arrest and lack of proliferation (CyclinA) in hepatocytes. G-CSF+TAK-242 mitigated senescence and significantly increased the rate of CyclinA expressing hepatocytes (figure) suggesting enhanced liver regeneration.

Conclusion The present study shows that G-CSF is deleterious in LPS-associated ACLF through further activation of inflammatory pathways and immune cell infiltration. TLR4 inhibition with TAK-242 prevented G-CSF driven tissue injury and induced liver regeneration showing evidence of synergy between the two molecules thereby providing a novel therapeutic strategy for ACLF patients.

\section{P094 POTENTIAL MECHANISMS UNDERLYING THE PROTECTIVE EFFECT OF LONG-TERM ALBUMIN INFUSION IN CIRRHOSIS}

\begin{abstract}
1,20ianwen Zhao, 'Alexandra Phillips*, 'Abeba Habtesion, 'Fausto Andreola, ${ }^{1}$ Nathan Davies, ${ }^{1} J a n e$ MacNaughtan, ${ }^{1}$ Rajiv Jalan. ${ }^{1}$ University College London, Institute for Liver and Digestive Health, London, UK; ${ }^{2}$ Department of Gastroenterology and Hepatology, West China Hospital, Sichuan University, China
\end{abstract}

\subsection{6/gutjnl-2021-BASL.102}

Background Long-term albumin administration has shown a reduction in mortality in hepatic decompensation, however the mechanism of this is unclear. The aims of this study were to determine (i) whether analbuminaemic (NAR) rats have greater liver injury and sensitivity to LPS (ii) whether albumin infusion is protective in NAR rats (iii) the impact of analbuminaemia and albumin infusion on the gut-liver interface and hepatic TLR4 signalling.

Methods 10 treatment groups of NAR and SD rats were studied $(n=4-7)$; Naïve, cirrhosis (4-w after bile duct ligation (BDL)) and ACLF models (induced by lipopolysaccharide (LPS) to BDL) were studied. BDL groups: \pm LPS, \pm albumin infusion (1.5 g/kg i.p. for 2 weeks). Markers of liver injury: plasma ALT level and TUNEL staining; markers of gut integrity and permeability: DAB immunohistochemistry ZO-1 expression in ileum tissue; Hepatic TLR4 immunohistochemistry and related pathway genes RT2 PCR profiler were studied.

Results Liver injury: ALT levels and TUNEL positive areas were significantly higher in NAR compared with SD rats $(\mathrm{p}=$ 0.01 and $\mathrm{p}=0.01)$, which were corrected with albumin infusion $(\mathrm{p}=0.02, \mathrm{p}=0.047)$. Effect of LPS administration: coma-free survival was higher in SD rats than NAR rats $(80 \%$ vs $40 \%$ ). Effect of albumin administration: Administration of albumin to BDL rats reduced severity of liver injury and mortality after LPS administration [p $=0.001 ; 40 \%$ vs $100 \%$, p $=0.04]$. Markers of gut permeability: In NAR rats, the histopathological examination of the ileum and colon revealed a reduction in ZO-1 expression, which was restored with 